\title{
The Internet, the Web, and Algorithms Invited Talk
}

\author{
Christos H. Papadimitriou \\ University of California at Berkeley, EECS \\ Berkeley, CA 94720 \\ christos@cs.berkeley.edu
}

The Internet and the worldwide web, unlike all other computational artifacts, were not deliberately designed by a single entity, but emerged from the complex interactions of many. As a result, they must be approached very much the same way that cells, galaxies or markets are studied in other sciences: By speculative (and falsifiable) theories trying to explain how selfish algorithmic actions could have led to what we observe. I present several instances of recent work on this theme, with several collaborators. 